\title{
Modelling loop-top X-ray source and reconnection outflows in solar flares with intense lasers
}

\author{
Jiayong Zhong ${ }^{1}$, Yutong $\mathrm{Li}^{2}$, Xiaogang Wang ${ }^{3}$, Jiaqi Wang ${ }^{3}$, Quanli Dong ${ }^{2}$, Chijie Xiao ${ }^{3}$, \\ Shoujun Wang ${ }^{2}$, Xun Liu ${ }^{2}$, Lei Zhang ${ }^{2}$, Lin An ${ }^{2}$, Feilu Wang ${ }^{1}$, Jianqiang Zhu ${ }^{4}$, Yuan Gu ${ }^{4}$, Xiantu He ${ }^{5,6,7}$, \\ Gang Zhao ${ }^{1 \star}$ and Jie Zhang ${ }^{2,8 \star}$
}

\begin{abstract}
Magnetic reconnection is a process by which oppositely directed magnetic field lines passing through a plasma undergo dramatic rearrangement, converting magnetic potential into kinetic energy and heat ${ }^{1,2}$. It is believed to play an important role in many plasma phenomena including solar flares ${ }^{3,4}$, star formation ${ }^{5}$ and other astrophysical events ${ }^{6}$, laser-driven

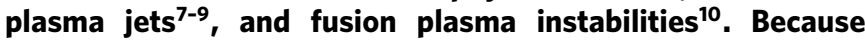
of the large differences of scale between laboratory and astrophysical plasmas, it is often difficult to extrapolate the reconnection phenomena studied in one environment to those observed in the other. In some cases, however, scaling laws ${ }^{11}$ do permit reliable connections to made, such as the experimental simulation of interactions between the solar wind and the Earth's magnetosphere ${ }^{12}$. Here we report well-scaled laboratory experiments that reproduce loop-top-like $X$-ray source emission by reconnection outflows interacting with a solid target. Our experiments exploit the mega-gauss-scale magnetic field generated by interaction of a high-intensity laser with a plasma to reconstruct a magnetic reconnection topology similar to that which occurs in solar flares. We also identify the separatrix and diffusion regions associated with reconnection in which ions become decoupled from electrons on a scale of the ion inertial length.
\end{abstract}

A major objective of laboratory astrophysics is to simulate the fundamental nature of astrophysical plasma physics processes in a laboratory environment so that certain astrophysical phenomenon can be studied in a controlled manner ${ }^{13}$. High energy density facilities, such as high-powered lasers and Z-pinches, can provide such opportunities $^{14}$, for example, direct measurements of opacity ${ }^{15}$, equations of state ${ }^{16}$, and photoionized plasmas ${ }^{17,18}$, as well as the similarity of physics, such as certain hydrodynamic phenomena of jets ${ }^{19}$ and shocks ${ }^{20}$ where a scaling law between astrophysical and laboratory plasma systems can be applied.

As a fundamental cause of many plasma energy conversion processes, magnetic reconnection (MR) is certainly a high priority of such studies. Masuda et al. ${ }^{21}$ observed the loop-top X-ray source in solar flares using the $\mathrm{YOHKOH}$ satellite and proposed that two antiparallel magnetic fields were merged above an arcade of closed loops as outflow jets from the reconnection point collided with high-density plasmas on the loop to produce a hot $\mathrm{X}$-ray region. Ultraviolet ${ }^{22}$ and $\mathrm{X}-\mathrm{ray}^{23,24}$ observations of plasma

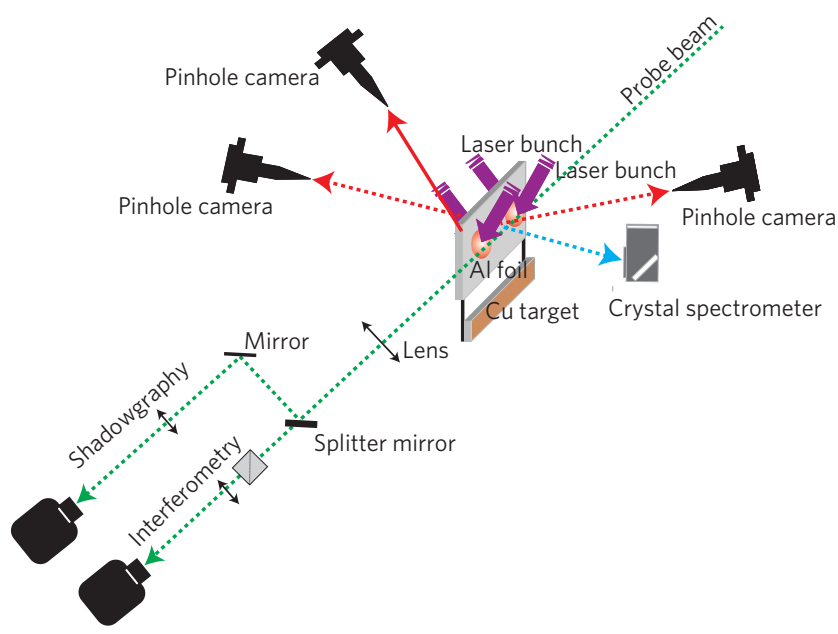

Figure 1 | Experimental set-up. Four bunches of long pulse (1 ns) lasers are focused on both sides of a thin Al foil target. Shadowgraphy and interferometry are used to diagnose the plasma evolution with a short pulse (120 ps) probe beam (shown as a green dotted line). The MR occurs between the two laser focus spots, and is detected by three $\mathrm{X}$-ray pinhole cameras. The reconnection outflow/jet can thus interact with a pre-set $\mathrm{Cu}$ target. X-ray spectra from the heated plasma are recorded by a crystal spectrometer set in front of the target.

jets ejected from the regions above the solar surface were also reported, and further confirm theoretical models of MR. Because of the great similarity of phenomena relating to MR in solar flares and laser-produced plasmas, here, by applying the scaling law of magnetohydrodynamics (MHD), we try to reproduce the reconnection outflow/jet and the loop-top X-ray source in the laboratory using a high-power laser facility.

Previous simulations and experiments ${ }^{7,8,25,26}$ showed that a mega-gauss (MG) magnetic field $\mathbf{B}$ could be generated in hot, high-density plasmas by irradiating a solid target using high-power laser beams. The B-field was approximately 'frozen' in the plasma bubbles. As the two bubbles expanded laterally and encountered each other with oppositely directed B-fields, MR occurred as the $\mathbf{B}$-field lines became topologically rearranged in the diffusion

\footnotetext{
${ }^{1}$ Key Laboratory of Optical Astronomy, National Astronomical Observatories, Chinese Academy of Sciences, Beijing 100012, China, ${ }^{2}$ Beijing National Laboratory for Condensed Matter Physics, Institute of Physics, Chinese Academy of Sciences, Beijing 100190, China, ${ }^{3}$ State Key Laboratory of Nuclear Physics \& Technology, School of Physics, Peking University, Beijing 100871, China, ${ }^{4}$ National Laboratory on High Power Lasers and Physics, Shanghai, 201800, China, ${ }^{5}$ Center for Applied Physics and Technology, Peking University, Beijing 100871, China, ${ }^{6}$ Institute for Fusion and Simulation, Zhejiang University, Hangzhou 310027, China, ${ }^{7}$ Institute of Applied Physics and Computational Mathematics, Beijing 100094, China, ${ }^{8}$ Department of Physics, Shanghai Jiao Tong University, Shanghai 200240, China. *e-mail: gzhao@bao.ac.cn; jzhang1@sjtu.edu.cn.
} 

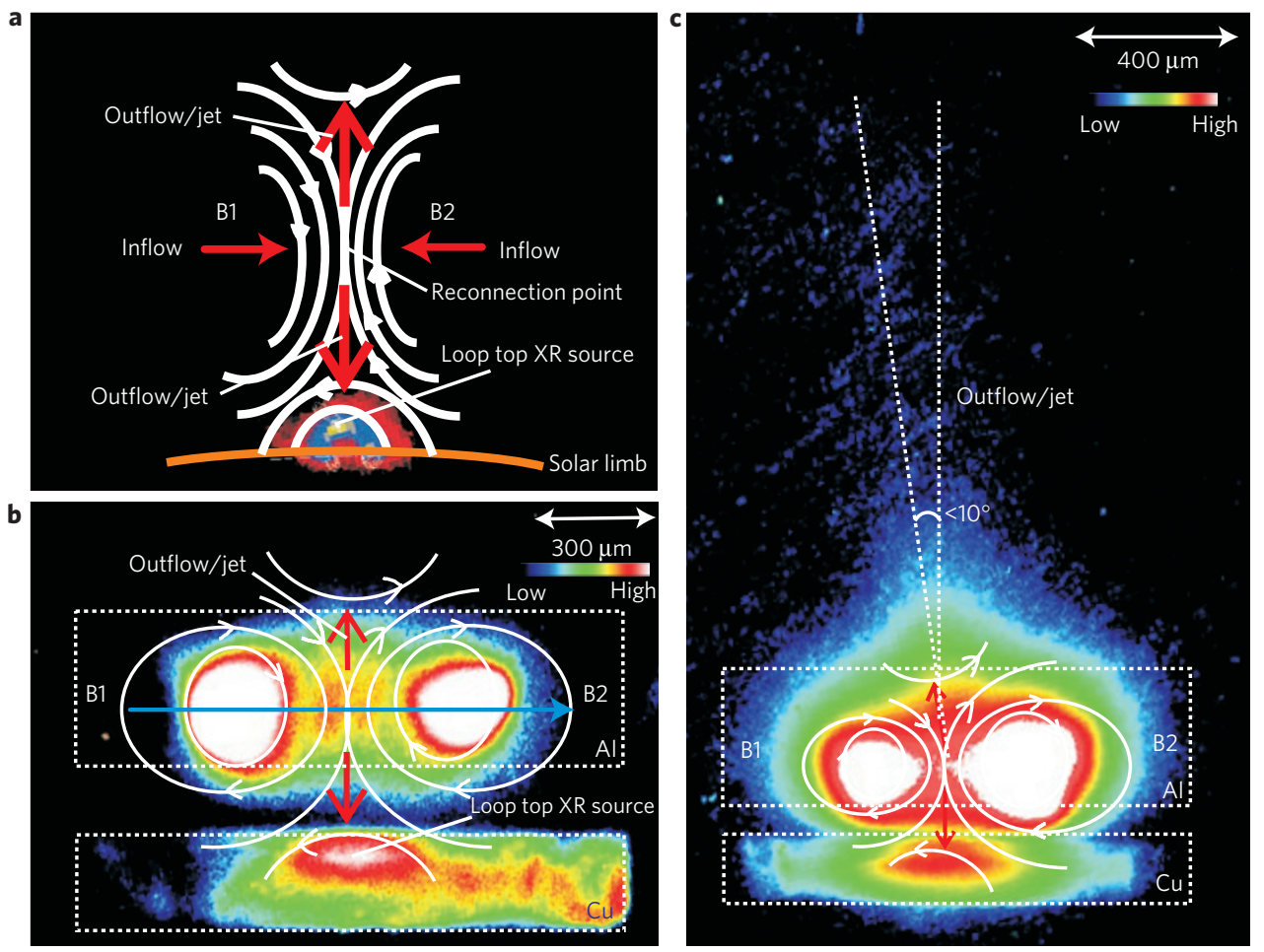

Figure 2 | Loop-top-like X-ray source and outflows observed in the laboratory. a, Magnetic reconnection model for the loop-top X-ray source in a compact solar flare, with a sketch depicting the X-ray observation scheme of ref. 21. b. The pinhole X-ray image observed forward of the Al foil target. Magnetic field lines are illustrated based on the flux surface of the plasma bubbles. The Al and Cu targets are the rectangles enclosed by white dotted lines. The red arrows indicate outflow/jet directions. c, X-ray image with two laser spots separated by $400 \mu \mathrm{m}$ and with a foil thickness of $10 \mu \mathrm{m}$. The asymmetry of the laser intensity on the Al target causes an imbalance of the laser spots as well as of the magnetic fields B1 and B2, and further induces the inclination of the upward outflow. The downward outflow impinges on the Cu target and results in a hot X-ray source.

Table 1 | The similarity of solar flares and laser-produced plasmas, with $a=10^{-11}, b=10^{8}, c=10^{10}$.

\begin{tabular}{llll} 
Parameters & For flare plasmas & For, & For flare plasmas (scaled) \\
\hline Length $(\mathrm{cm})$ & $\sim 10^{9}-10^{10}$ & $\sim 10^{-1}$ & $\sim 10^{-2}-10^{-1}$ \\
Time $(\mathrm{s})$ & $\sim 100-1,000$ & $\sim 10^{-9}$ & $\sim 10^{-9}-10^{-10}$ \\
Pressure $(\mathrm{Pa})$ & $\sim 0.001-10$ & $\sim 10^{7}$ & $\sim 10^{7}-10^{11}$ \\
Density $\left(\mathrm{cm}^{-3}\right)$ & $\sim 10^{9}-10^{11}$ & $\sim 10^{19}-10^{20}$ & $\sim 10^{19}-10^{21}$ \\
Velocity $\left(\mathrm{km} \mathrm{s}^{-1}\right)$ & $\sim 10-100$ & $\sim 100$ & $\sim 100-1,000$ \\
Magnetic field (G) & $\sim 10-100$ & $\sim 10^{6}$ & $\sim 10^{6}-10^{7}$
\end{tabular}

region. It thus enabled us to simulate the solar flare loop-top X-ray source generation process in the laboratory.

The experiment was performed at the Shenguang (SG) II laser facility, which can deliver a total energy of $2.0 \mathrm{~kJ}$ in a nanosecond square pulse. The eight SG II laser beams, with a wavelength of $\lambda_{\mathrm{L}}=0.351 \mu \mathrm{m}$, are divided into four bunches. Each bunch then consists of two laser beams. The geometric configuration, as shown in Fig. 1, is designed to be similar to the scheme of a loop-top X-ray source in the solar flares depicted in Fig. 2a. Two synchronized laser bunches separated by $400-600 \mu \mathrm{m}$ are focused onto one side of the $\mathrm{Al}$ foil with the other two laser bunches symmetrically irradiating the other side simultaneously. Each bunch is focused to a focal spot diameter of 50-100 $\mu \mathrm{m}$ full width at half maximum (FWHM), giving an incident laser intensity of $\sim 5 \times 10^{15} \mathrm{~W} \mathrm{~cm}^{-2}$. $\mathrm{A} \mathrm{Cu}$ target is set $250 \mu \mathrm{m}$ away from one foil edge. The $\mathrm{Al}$ foil is $1,600 \mu \mathrm{m} \times 500 \mu \mathrm{m}$ with a thickness of $10-50 \mu \mathrm{m}$. The $\mathrm{Cu}$ target is $1,600 \mu \mathrm{m} \times 250 \mu \mathrm{m}$ with a thickness of $150 \mu \mathrm{m}$. The X-ray emission is measured using three $\mathrm{X}$-ray pinhole cameras in the forward, side and reverse directions, to investigate the reconnection jets as well as their impact on the copper target. The image is taken through a $10 \mu \mathrm{m}$ pinhole, filtered with $50 \mu \mathrm{m}$ of beryllium, allowing all X-rays above $\sim 1 \mathrm{keV}$ to pass. Most of the signal from the high-energy continuum is recorded using time-integration on an X-ray film with its highest sensitivity to $\mathrm{X}$-rays in the $1-10 \mathrm{keV}$ range. A flat crystal spectrometer is set in front of the targets to record the X-ray spectra from the heated plasmas. Shadowgraphy and interferometry with a $120 \mathrm{ps}$ green $\left(\lambda_{\mathrm{L}}=0.53 \mu \mathrm{m}\right)$ laser beam are also used to investigate the evolution of the plasma.

The process can be reasonably described by MHD, as the magnetic Reynolds number is very high. Ryutov et al. ${ }^{13}$ demonstrated the scaling relations of two ideal MHD systems $\left(\operatorname{Re}_{M} \gg 1\right)$, in which the variables of the systems remain invariant under such transformations, as $r=a r_{1}, \rho=b \rho_{1}, p=c p_{1}, t=a \sqrt{b / c} t_{1}$, $v=\sqrt{c / b} v_{1}, B=\sqrt{c} B_{1}$, where $r$ is the characteristic length, $\rho$ is the mass density, $p$ is the pressure, $v$ is the velocity, $B$ is the magnetic field of the systems, and $a, b, c$ are transformation coefficients. By choosing laser parameters and target materials properly, the magnetic Reynolds number $\operatorname{Re}_{\mathrm{M}} \approx 0.8 \sqrt{(Z+1) / Z^{2} A} L(\mathrm{~cm})(T(\mathrm{eV}))^{2}$ is 


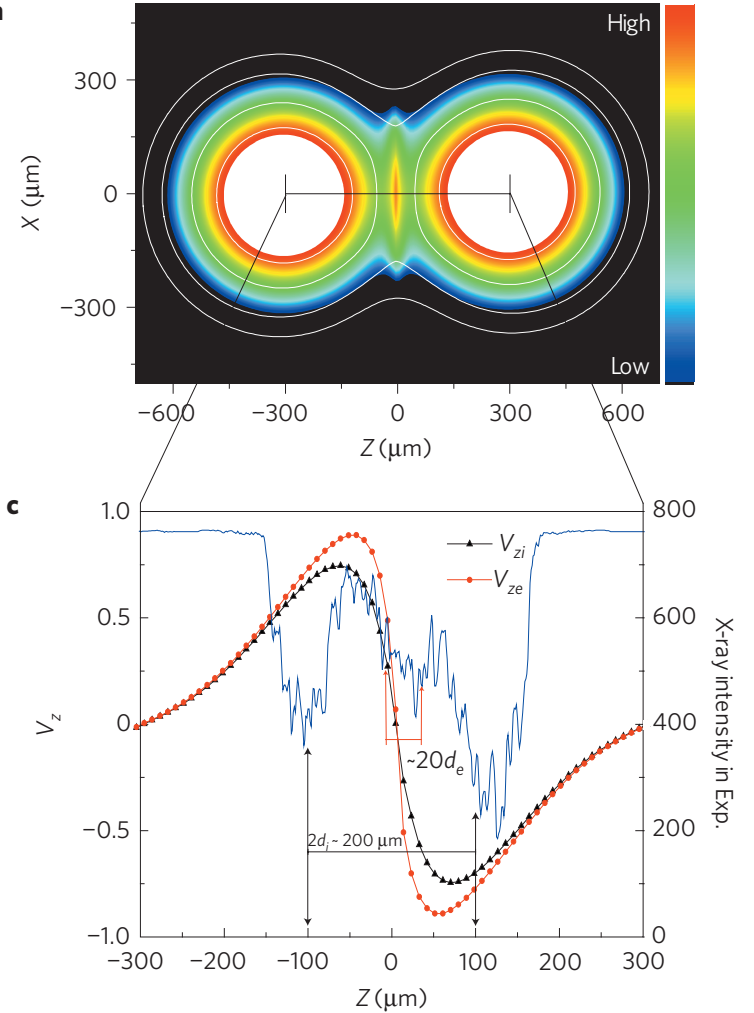

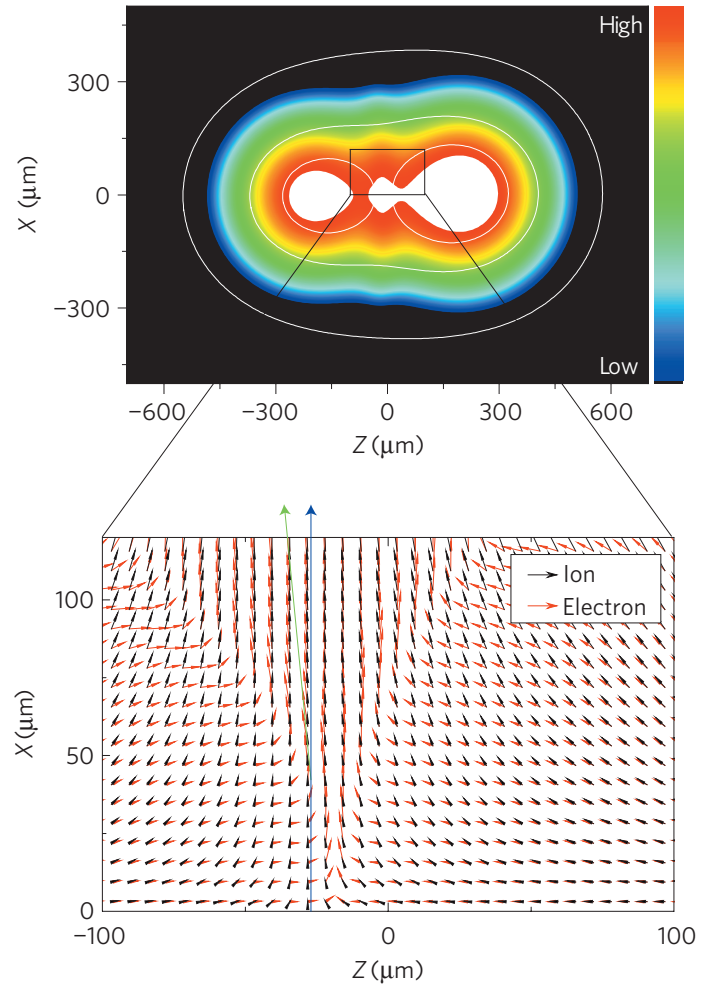

Figure 3 | Simulation results with Hall MHD code. $\mathbf{a}, \mathbf{b}, X$-ray images of the numerical MR simulation corresponding to the experiments of Fig. $2 \mathrm{~b}$ and $\mathrm{c}$, respectively. $\mathbf{c}$, The inflow velocity $V_{z}$ (black triangle for ions and red dot for electrons), together with the one-dimensional cross-section of an $X$-ray image between the two laser spots in Fig. $2 \mathrm{~b}$ (blue solid line). The distance between the two X-ray patterns denoted by black arrows is on the order of the ion inertial length $2 d_{i} \approx 200 \mu \mathrm{m}$, comparable to the size of the ion-electron decoupling region. The interface between the two X-ray patterns, about $20 d_{e} \approx 40 \mu \mathrm{m}$, is denoted by red arrows. $\mathbf{d}$, The vector velocity of ion (black arrow) and electron (red arrow) flows plotted for the area enlarged from $\mathbf{b}$. The inclination is shown to be in agreement with the experimental result in Fig. 2c.

around 4,000 (for $Z=13, A=27, L=0.1 \mathrm{~cm}, T=1,000 \mathrm{eV}$ ), which makes the MHD processes in laser plasmas and solar flares $\left(\operatorname{Re}_{\mathrm{M}} \sim 5 \times 10^{8}\right)$ comparable.

The similarity of the MHD in solar flares and laser-produced plasmas is shown in Table 1, with the transformation coefficients $a=10^{-11}, b=10^{8}$, and $c=10^{10}$. The scaled parameters of the solar coronal plasmas in the third column are very similar to those of the laser-produced plasmas in the second column.

Two bright X-ray spots are clearly observed resulting from the laser heating the Al foil target. In Fig. $2 \mathrm{~b}$, the two laser spots are separated by $600 \mu \mathrm{m}$, nearly 6-7 laser focus diameters, in vacuum to reproduce a previously studied geometry of laser driven magnetic reconnection ${ }^{8}$. The spontaneous magnetic field has an estimated MG strength based on hydrodynamic simulations and similar experimental measurements ${ }^{7,8}$. When two plasma bubbles expand on the Al foil surface, two toroidal MG magnetic fields 'frozen' in the bubbles merge accordingly with each other. The breakdown of the 'frozen in' condition occurs when the oppositely directed fields B1 and B2 encounter each other between the spots, where a diffusion region can be clearly seen with two significant $\mathrm{X}$-ray patterns showing the release of magnetic energy. The width across each pattern is on the order of the ion inertial length $c / \omega_{\mathrm{pi}}=2.28 \times 10^{7} z^{-1}\left(\mu / n_{i}\right)^{1 / 2}(\mathrm{~cm}) \approx 100 \mu \mathrm{m}$, with an ion density of $10^{18} \mathrm{~cm}^{-3}$. In this region electrons and ions are decoupled, and MR occurs. Note that there is also a clear interface between the two $\mathrm{X}$-ray emission patterns, which is possibly a magnetic separatrix between two plasmas with the dominant magnetic field component tangential to it. A similar experiment, however, was carried out with two imbalanced laser beams separated by $400 \mu \mathrm{m}$, as shown in Fig. 2c. Consequently, the upflow is not vertical but has an inclination of $\sim 10^{\circ}$, providing an interpretation for the plasma jet inclination in solar flares. The X-ray intensity, on the other hand, is greatly enhanced in comparison with that in Fig. $2 \mathrm{~b}$ as a result of the small separation. The diffusion region can hardly be defined here because the emission in the region is too high to distinguish from the spots.

The most striking feature in both experiments is that a bright $\mathrm{X}$-ray spot at the centre of the $\mathrm{Cu}$ target is observed just below the downward outflow/jet. The position and the arc shape of the spot is solid evidence that there is a high-speed outflow/jet on the $\mathrm{Al}$ foil impacting the plasma generated on the $\mathrm{Cu}$ target, a picture clearly resembling the loop-top X-ray source in solar flare observations. In Fig. 2c, the upflow ejected out of the $\mathrm{Al}$ target due to MR can also clearly be seen. It is more than $2 \mathrm{~mm}$ with a width of $300 \mu \mathrm{m}$, or $2 \times 10^{5} \mathrm{~km}$ with width of $3 \times 10^{4} \mathrm{~km}$ when scaled for a solar plasma; this is on the order of the typical lengths and widths of X-ray jets observed in solar flares ${ }^{23}$. The flow velocity was measured to be $400( \pm 50) \mathrm{km} \mathrm{s}^{-1}$ from time-resolved shadowgrams in the laboratory, which agrees well with the typical Alfvén speed of $V_{\mathrm{A}} \approx 400 \mathrm{~km} \mathrm{~s}^{-1}$, in a magnetic field of $10^{6} \mathrm{G}$ for the experiment as well as the transverse velocity for bi-directional plasma jets of $\sim 150-300 \mathrm{~km} \mathrm{~s}^{-1}$ scaled from observations ${ }^{22}$. Here we assume the scaling law is valid during the whole reconnection process.

For the experiment, the initial state is not in equilibrium. On the edge of the plasma, the force balance is broken down because of an imbalance between the pressure gradient and the Lorentz force. Therefore, the reconnection process is extremely strongly driven. Next, we numerically simulated the experiment with a twodimensional/3-component Hall MHD code, for the balanced beams with a $600 \mu \mathrm{m}$ separation (Case 1), and for the imbalanced beams 
with a $400 \mu \mathrm{m}$ separation (Case 2 ). The X-ray emission obtained in both the simulations agrees well with experimental results, as shown in Fig. 3a, and b. The reconnection geometry is found to be 'Y-type' (Fig. 3d), justifying the Sweet-Parker geometry, and the decoupling of ion and electron flows is also clearly shown by the black arrows (Fig. 3c) in the region with the ion inertia scale of $d_{i}=c / \omega_{\mathrm{pi}} \approx 100 \mu \mathrm{m}$, which is in good agreement with the experiment. A one dimensional cross section of the experimentally observed X-ray images between the two focal spots is also plotted for comparison to the numerical result. It can be seen that the $\mathrm{X}$-ray signal patterns in the regions denoted by black arrows are also where the ion and electron flows are decoupled most significantly in the numerical simulation. The interface between the two X-ray peaks illustrated with red arrows shows a possible electron diffusion region around $10 c / \omega_{\text {pe }}$, larger than the theoretical prediction but in a good agreement with a recent MRX measurement ${ }^{29}$. We also find that reconnection is much faster $(t=0.8 \mathrm{~ns})$ than typical SweetParker resistive reconnection, clear evidence of fast reconnection due to both the Hall effect and the boundary conditions ${ }^{30}$. The simulation of the imbalanced Case 2, as shown in Fig. $3 \mathrm{~b}$ and d, also reproduced the experimental result in Fig. 2c well.

The experiment reported here is the first laboratory simulation of a MR induced loop-top X-ray source and outflow/jet with highpower lasers. MG magnetic fields and high-energy-density plasmas generated by intense laser pulses allow us to study astrophysical MR on a laboratory scale. Such measurements with the controlled parameters of laser-produced plasmas should greatly benefit the understanding of not only explosive energy release and particle acceleration processes such as solar flares, but also many other astrophysical phenomena related to MR.

Received 15 March 2010; accepted 17 August 2010; published online 10 October 2010; corrected online 11 October 2010

\section{References}

1. Yamada, M. Progress in understanding magnetic reconnection in laboratory and space astrophysical plasmas. Phys. Plasmas 14, 058102 (2007).

2. Zweibel, E. \& Yamada, M. Magnetic reconnection in astrophysical and laboratory plasmas. Annu. Rev. Astron. Astrophys. 47, 291-332 (2009).

3. Sweet, P. A. in The Neutral Point Theory of Solar Flares, IAUsymp.6 (ed. Lehnert, B.) 123 (Cambridge Univ. Press, 1958).

4. Parker, E. N. Sweet's mechanism for merging magnetic fields in conducting fluids. J. Geophys. Res. 62, 509 (1957).

5. Kulsrud, R. M. Magnetic reconnection in a magnetohydrodynamic plasma. Phys. Plasmas 5, 1599 (1998).

6. Goodman, J. \& Uzdensky, D. Reconnection in marginally collisionless accretion disk coronae. Astrophys. J. 688, 555-558 (2008).

7. Nilson, P. M. et al. Magnetic reconnection and plasma dynamics in two-beam laser-solid interactions. Phys. Rev. Lett. 97, 255001 (2006).

8. Nilson, P. M. et al. Bidirectional jet formation during driven magnetic reconnection in two-beam laser-plasma interactions. Phys. Plasmas 15, 092701 (2008).

9. Li, C. K. et al. Observations of electromagnetic fields and plasma flow in hohlraums with proton radiography. Phys. Rev. Lett. 102, 205001 (2009).

10. Taylor, J. B. Relaxation and magnetic reconnection in plasmas. Rev. Mod. Phys. 58, 741-763 (1986).

11. Ryutov, D. D., Drake, R. P. \& Remington, B. A. Criteria for scaled laboratory simulations of astrophysical MHD phenomena. Astrophys. J. 127 (suppl.), 465-468 (2000).
12. Brady, P., Ditmire, T., Horton, W., Mays, M. L. \& Zakharov, Y. Laboratory experiments simulating solar wind driven magnetospheres. Phys. Plasmas 16, 043112 (2009).

13. Remington, B. A., Drake, R. P. \& Ryutov, D. D. Experimental astrophysics with high power lasers and Z pinches. Rev. Mod. Phys. 78, 755-807 (2006).

14. Takabe, H. Astrophysics with intense and ultra-intense lasers "laser astrophysics". Prog. Theor. Phys. Suppl. 143, 202-265 (2001).

15. Rogers, F. J. \& Iglesias, C. A. Astrophysical opacity. Science 263, 50-55 (1994).

16. Celliers, P. M. et al. Electronic conduction in shock-compressed water. Phys. Plasmas 11, L41-L44 (2004)

17. Foord, M. E. et al. Charge-state distribution and Doppler effect in an expanding photoionized plasma. Phys. Rev. Lett. 93, 055002 (2004).

18. Fujioka, S. et al. X-ray astronomy in the laboratory with a miniature compact object produced by laser-driven implosion. Nature Phys. 11, 495-497 (2009).

19. Gregory, C. D. et al. Astrophysical jet experiments with colliding laser-produced plasmas. Astrophys. J. 676, 420-426 (2008).

20. Foster, J. M. et al. Supersonic jet and shock interactions. Phys. Plasmas $\mathbf{9}$, 2251-2263 (2002)

21. Masuda, S. et al. A loop-top hard X-ray source in a compact solar flare as evidence for magnetic reconnection. Nature 371, 495-497 (1994).

22. Innes, D. E., Inhester, B., Axford, W. I. \& Wilhelm, K. Bi-directional plasma jets produced by magnetic reconnection on the Sun. Nature 386, 811-813 (1997).

23. Cirtain, J. W. et al. Evidence for Alfvén waves in solar X-ray jets. Science 318, 1580-1582 (2007).

24. Shibata, K. et al. Chromospheric anemone jets as evidence of ubiquitous reconnection. Science 318, 1591-1594 (2007).

25. Yates, M. A., van Hulsteyn, D. B., Rutkowski, H., Kyrala, G. \& Brackbill, J. U. Experimental evidence for self-generated magnetic fields and remote energy deposition in laser-irradiated targets. Phys. Rev. Lett. 49, 1702-1704 (1982).

26. Li, C. K. et al. Observation of megagauss-field topology changes due to magnetic reconnection in laser-produced plasmas. Phys. Rev. Lett. 99, 055001 (2007).

27. Bray, R. J., Cram, L. E., Durrant, C. \& Loughhead, R. E. Plasma Loops in the Solar Corona (Cambridge Univ. Press, 1991).

28. Lin, J. et al. Features and properties of coronal mass ejection/flare current sheets. Astrophys. J. 658, L123-L126 (2007).

29. Ren, Y., Yamada, M., Ji, H., Gerhardt, S. \& Kulsrud, R. Identification of the electron-diffusion region during magnetic reconnection in a laboratory plasma. Phys. Rev. Lett. 101, 085003 (2008).

30. Wang, X., Bhattacharjee, A. \& Ma, Z. W. Scaling of collisionless forced reconnection. Phys. Rev. Lett. 87, 265003 (2001).

\section{Acknowledgements}

We would like to acknowledge the Shenguang II staff for operating the laser facility, CAEP staff for providing some diagnostics and target fabrication. We also thank J. Lin, Y. Zhang, and J. Wang from NAOC for valuable discussions. This work was supported by the National Basic Research Program of China (973 Program) (Grant Nos. 2007CB815100, 2009GB105004, and 2006CB806300), and the National Natural Science Foundation of China (Grant Nos. 10821061, 10925421, 10734130, 40731056, 40974104, and 10975012)

\section{Author contributions}

J.Y.Z. proposed the experiment. Q.D. and Y.L. were in charge of the experiment campaign. The experimental data were measured and analyzed by J.Y.Z., Q.D., S.W., X.L., L.Z., L.A. and Y.L. The theoretical analysis was carried out by X.W., J.W., J.Y.Z., C.X. and F.W. X.H. was involved the early part of the discussion. J.Q.Z., Y.G. and their colleagues are responsible for running the laser facility and target area. J.Y.Z., Y.L. and X.W. contributed to writing of the manuscript. J.Z. and G.Z. are the proposers and principal investigators of the laboratory astrophysics project.

\section{Additional information}

The authors declare no competing financial interests. Reprints and permissions information is available online at http://npg.nature.com/reprintsandpermissions. Correspondence and requests for materials should be addressed to G.Z. or J.Z. 